404. H. V. Pechmann: Ueber Diazomethandisulfonsäure.

[2. Mittheilung ${ }^{1}$ ].

[Aus dem chemischen Laboratorium der Universitāt zu Tübingen.]

(Eingeg. am 15. August.)

Verhalten beim Erhitzen.

Es ist eine schon früber erwäbnte charakteristiscbe Eigenthümlichkeit des diazomethandisulfonsauren Kaliums, beim Erhitzen nach dem Verlust des Krystallwassers stickstoffärmere Verbindungen zu liefern. Eine derselben ist unschwer zu isoliren. Sie entstebt nach der Gleichung:

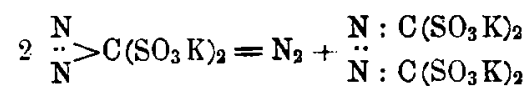

und soll als azinmethandisulfonsaures Kalium bezeichnet werden. Diese Reaction entspricht dem von Curtius ${ }^{2}$ ) beschriebenen Uebergang der Diazobernsteinsäure in die unsymmetrische Azinberngteinsäure und darf wobl als ein weiterer Beweis für die Richtigkeit der Auffassung der Diazometbandisulfonsäure gelten.

\title{
Azinmethandisulfonsaures Kalium
}

wird dargestellt, indem man das zcrriebene Diazosalz zuerst bei $100^{\circ}$ im Wasserstoffstrom entwässert und dann $10-12$ Stunden auf $120-130^{\circ}$ erhitzt, und zwar, um sonst mögliche Explosionen zu vermeiden, am zweckmässigsten durch Kochen mit Xylol. Das scbwach gefärbte, meist noch Diazosalz entbaltende Reuctionsproduct geht beim Kochen mit der fünffachen Menge Wasser unter lebbafter Gasentwicklung in Lösung. Beim Erkalten krystallisirt die neue Verbindung in weissen rosetten- oder sägeförmig gruppirten Prismen aus. Ausbeute $50 \mathrm{pCr}$. des Ausgangsmaterials.

Analyse: Ber. für $\mathrm{C}_{2} \mathrm{~N}_{2}\left(\mathrm{SO}_{3} \mathrm{~K}\right)_{4}, 2 \mathrm{H}_{2} \mathrm{O}$.

$$
\begin{aligned}
& \text { Proc.: C 4.3, } \mathrm{H} \text { 0.7, N 5.0, S 23.9, K 27.9. } \\
& \text { Gef. 》 » } 4.2,3.6, » 1.2,0.9, » 4.5, » 23.2 \text {, 》27.7. }
\end{aligned}
$$

Die Krystallwasserbestimmungen:

$$
\begin{aligned}
& \text { Ber. Procente: } \mathrm{H}_{2} \mathrm{O} 6.4 . \\
& \text { Gef. } \quad 7 \quad 7.0-7.4 \text {. }
\end{aligned}
$$

lieferten etwas zu bohe Zablen, weil die Verbindung bei höherer Temperatur ausser Wasser auch Stickstoff abgiebt. Ein analysenreines weiteres Abbauproduct ist noch nicht isolirt worden.

Das Verbalten der Verbindung gegen kochende Säuren entspricht der gegebenen Formel; der Schwefel wird als schweflige Säure, der Stickstoff als Hydrazin abgespalten. Charakteristisch verbält sie sich gegen Chlorbaryum, wodnrch in mässig verdünnter Lösung ein Niederschlag entsteht, der im Ueberschuss des Fällungs-

1) Vergl. diese Berichte 28, 2374.

2) Journ. f, prakt. Chem. 39, 135. 
mittels wieder verschwindet, worauf alsbald glänzende zugespitzte Blättchen auskrystallisiren, die sich auch beim Kochen nicht mehr Jösen. Eine bemerkenswerthe Veränderung, welche durch Kalilauge hervorgerufen wird, ist bei der Schwierigkeit, grössere Mengen der Verbindung darzustellen, noch nicht studirt worden.

Verhalten gegen Diazobenzol.

Sowohl diazomethandisulfonsaures Kalium ais Diazoessigester vereinigen sich mit Kaliumsulfit zu krystallisirenden Additionsproducten, welche als sulfohydrazimethylendisulfonsares Kalium $\left.{ }^{1}\right),\left(\mathrm{SO}_{3} \mathrm{~K}\right)_{2} \mathrm{CH}<\underset{\mathrm{NSO}_{3} \mathrm{~K}}{\mathrm{NH}}$, und $\mathrm{Kaliumsulfobydrazimethylen-}$ carbonester ${ }^{2}$ ), $\operatorname{COOC}_{2} \mathrm{H}_{5} . \mathrm{C}<\underset{\mathrm{N} \mathrm{NO}_{3} \mathrm{~K}}{\mathrm{NH}}$, beschrieben worden sind. Die Berechtigung dieser Formeln beruht anf der Annahme, dass die Addition ron Sulfit an aliphatische Diazorerbindungen in derselben Weise wie in der aromatiscben Reibe ${ }^{3}$ ) verläuft, nämlich einfach unter Lösung der doppelten Stickstnff bindung. Einwurfsfrei waren sie vielleicht deshalb uicht, weil unter Sprengung des Diazomethanringes auch Hydrazone entstehen konuten, z. B.

$\mathrm{COOC}_{2} \mathrm{~B}_{5} . \mathrm{CH}<\underset{\mathrm{N}}{\mathrm{N}}+\mathrm{SO}_{3} \mathrm{~K}_{2}+\mathrm{H}_{2} \mathrm{O}=\mathrm{COOC}_{2} \mathrm{H}_{5} . \mathrm{C}: \mathrm{N} . \mathrm{NHSO}_{3} \mathrm{~K}+\mathrm{KOH}$.

Die Wahrnehmung, dass die oben erwäbuten Sulfitverbindungen mit Diazobenzol reagiren, fübrte zu einer Reihe ron Beobachtungen, welche die bisberigen Formeln bestätiagen dürften.

\section{Sulfitverbindung des Diazoessigesters und Diazobenzol.}

Diese Verbiudungen liefern in essigsaurer Lösung neben Stickstoff and Schwefelsäure das Phenylhydrazon des Glyoxylsäureesters nach der Gleichung:

$\left.\mathrm{COOC}_{2} \mathrm{H}_{5} \cdot \mathrm{CH}<\underset{\mathrm{NSO}_{3} \mathrm{~K}}{\mathrm{NH}}+\mathrm{NO} \cdot \mathrm{NHC}_{6} \mathrm{H}_{5}{ }^{4}\right)=\mathrm{COOC}_{2} \mathrm{H}_{5} \cdot \mathrm{CH}: \mathrm{N} . \mathrm{NHC}_{6} \mathrm{H}_{5}$

$$
+\mathrm{N}_{2}+\mathrm{SO}_{4} \mathrm{HK} \text {. }
$$

In alkalischer Lösung geht die Raaction weiter, indem unter Aufuahme eines zweiten Diazobenzolmoleküls Formazylameisenester entsteht. Wäre die Sulfitverbindung des Diazoessigesters ein Hydrazon, so bätte schon die Aufnahme des ersten Diazobenzols einen Formazylkörper liefern sollen: $\mathrm{COOC}_{2} \mathrm{H}_{5}$. CH : $\mathrm{N} . \mathrm{NHSO}_{3} \mathrm{~K}+\mathrm{HON}: \mathrm{NC}_{6} \mathrm{H}_{5}$

was nicht der Fall ist.

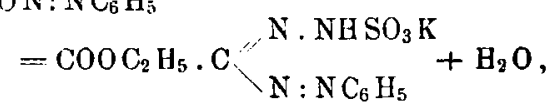

1) v. Pechmann und Manck, diese Berichte 28, 2380.

3) Diese Berichte 28, $3847 . \quad 3$ 3) Diese Berichte 28, 863.

4) Die Nitrosaminformel gestititet die einfachste Formulirung. 
Phenylhydrazonglyoxylsäureester

entsteht als gelblicher Niederschlag, wenn in die auf $0^{0}$ abgekũhlte Lösung der Sulfitverbindung schwach saures Diazobenzolacetat einfliesst. Aus verdünntem Alkohol krystallisirt das Hydrazon in fast farblosen rhombischen Blättchen. Ausbeute quantitativ.

Analyse: Ber. für $\mathrm{C}_{6} \mathrm{H}_{5} \mathrm{NH} . \mathrm{N}: \mathrm{CH} . \mathrm{COOC}_{2} \mathrm{H}_{5}$.

$$
\begin{array}{ll}
\text { Procente: N } 17.04 . \\
\text { Gef. } & 16.84 .
\end{array}
$$

Schmp. 130-1310. Löslich in den meisten beissen Lösungsmitteln. Ferrichlorid oder Kaliumdichromat färben die gelbe Lösung in conc. Schwefelsäure rothviolet. Mit alkoholischem Natron verseift, entsteht das bekannte Phenylhydrazon der Glyoxylsäure, das bei $143-145^{\circ}$, also etwas böher als nach den bisherigen Angaben schmilzt.

\section{Formazylameisenester}

erbält man als tiefrothe Färbung, wenn die Lösung der Sulfitverbindung in die auf $0^{0}$ abgekühlte alkalische Lösung von 2 Mol. Diazobenzol gegossen wird. Ausbeute $80 \mathrm{pCt}$. der Theorie. Krystallisirt aus kochendem Alkohol in den charakteristischen Schuppen rom Schmp. 1170. Beim Verseifen entsteht Formazylameisensäure vom Schmp. $162^{\circ}$.

\section{Sulfitverbindung des diazomethandisulfonsauren Kaliums und Diazobenzol.}

In essigsaurer Lösung verläuft die Reaction wie bei dem Diazoessigesterderirat. Unter Elimination des Stickstoffs und der Sulfogruppe als Schwefelsäure entsteht das Phenylhydrazon der Methanaldisulfonsäure:

$$
\begin{aligned}
\left\langle\mathrm{SO}_{3} \mathrm{~K}\right)_{2} \mathrm{C}< & \stackrel{\mathrm{NH}}{\mathrm{N}} \stackrel{\mathrm{NSO}}{3} \mathrm{~K}^{\mathrm{H}}+\mathrm{NO} . \mathrm{NHC}_{6} \mathrm{H}_{5} \\
= & \left(\mathrm{SO}_{3} \mathrm{~K}\right)_{2} \mathrm{C}: \mathrm{N} . \mathrm{NHC}_{6} \mathrm{H}_{3}+\mathrm{N}_{2}+\mathrm{SO}_{4} \mathrm{KH},
\end{aligned}
$$

welches im Kleinen auch aus Methandisulfonsäurester und alkalischem Diazobenzol erhalten wurde.

Es wird dadurch sebr unwabrscheinlich, dass das Sulfitderivat der Diazoverbindung ein Hydrazon ist, denn ein solches hätte eine Formazylverbindung liefern sollen. Eine solche entsteht aber zunächst nicht einmal in alkalischer Lösung; in diesem Falle vehmen wiederum zwei Moleküle Diazobenzol an der Reaction Theil, wobei das primär entstandene Hydrazon, an dessen Hydrazonkoblenstoff kein bewegliches Wasserstoffatom haftet, in einen diazoamidobenzolartigen Körper, diazobenzolphenyl bydrazonmethandisulfonsaures Kalium, übergeht:

$$
\begin{aligned}
\left(\mathrm{SO}_{3} \mathrm{~K}\right)_{2} \mathrm{C}: \mathrm{N} . \mathrm{NHC} \mathrm{C}_{6} \mathrm{H}_{3} & +\mathrm{HON}: \mathrm{NC}_{6} \mathrm{H}_{5} \\
& =\left(\mathrm{SO}_{3} \mathrm{~K}\right)_{2} \mathrm{C}: \mathrm{N} \cdot \mathrm{N}\left(\mathrm{C}_{6} \mathrm{H}_{5}\right) \cdot \mathrm{N}: \mathrm{NC}_{6} \mathrm{H}_{5}+\mathrm{H}_{2} \mathrm{O} .
\end{aligned}
$$

Rerichte d, D. chem. Gesel!schaft. Jahrg. XXIX. 
Die Verbindung entsteht auch durch alkaliscbe Kupplung des Hydrazons mit Diazobenzol. Als Diazoamidoverbindung erleidet sie schon durch verdünnte kalte Säuren Spaltung in Diazobenzol und Hydrazon. Alkoboliscbe Sulzsäure lagert sie dagegen in die freie Formazylsulfonsäure um unter Abspaltung einer Sulfogruppe:

$\left(\mathrm{SO}_{3} \mathrm{H}\right)_{2} \mathrm{C}: \mathrm{N} \cdot \mathrm{N}\left(\mathrm{C}_{6} \mathrm{H}_{5}\right) \cdot \mathrm{N}: \mathrm{NC}_{6} \mathrm{H}_{5}+\mathrm{H}_{3} \mathrm{O}$

$$
=\mathrm{SO}_{3} \mathrm{H} . \mathrm{C} \backslash \mathrm{N}: \mathrm{NC}_{6} \mathrm{H}_{5}+\mathrm{SO}_{4} \mathrm{H}_{2} \text {. }
$$

Dass die neue Formazylverbindung nicht das Product einer complicirten Reaction, sondern einer einfachen Umlagerung ist, dürfte darans bervorgehen, dass die aus dem Hydrazon und Bromdiazobenzol entstebende Monobromdiazoamidoverbindung durch alkoholische Salzsäure in eine Monobromformazyl sulfons ä u re verwandelt wird.

Für die Richtigkeit der Auffassung der Formazylsulfonsäure spricht ihr Verbalten bei der Reduction mit Zinkstaub und verdünnten Säuren. H. v. Pechmann und P. Runge') haben gezeigt, dass Formazylverbindungen dabei in Hydrazin und Hydrazid gespalten werden. Die Formazylsulfonsäure zeigt ein analoges Verhalten gemäss der Gleichung:

$$
\begin{gathered}
\mathrm{SO}_{3} \mathrm{H} . \mathrm{C} \backslash \mathrm{N}: \mathrm{NC}_{6} \mathrm{H}_{5} \\
\quad=\mathrm{SO}_{3} \mathrm{H} . \mathrm{CO} \cdot \mathrm{NH} . \mathrm{NHC} \mathrm{H}_{6} \mathrm{H}_{5}+\mathrm{NH}_{2} \cdot \mathrm{NHC}_{6} \mathrm{H}_{5} .
\end{gathered}
$$

Jene Sulfonsäure erleidet sofort eine weitere Veränderung, indem sie theils nach der Gleichung:

$\mathrm{SO}_{3} \mathrm{~B} . \mathrm{CO}$. $\mathrm{NH}$. $\mathrm{NHC} \mathrm{C}_{6} \mathrm{~B}_{5}+\mathrm{H}_{2} \mathrm{O}=\mathrm{SO}_{3} \mathrm{H}_{2}+\mathrm{CO}_{2}+\mathrm{NH}_{2}$. NH. $\mathrm{C}_{6} \mathrm{H}_{5}$ zerfällt, theils unter Addition der so entstandenen schwefligen Säure $\mathrm{Phenylhydrazonmethandisulf} \mathrm{ousäure} \mathrm{liefert} \mathrm{nach} \mathrm{der} \mathrm{Gleichung:}$

$\mathrm{SO}_{3} \mathrm{H}$. CO . NH . NH $\mathrm{C}_{6} \mathrm{H}_{5}+\mathrm{SO}_{3} \mathrm{H}_{2}=\left(\mathrm{SO}_{3} \mathrm{H}\right)_{3} \mathrm{C}: \mathrm{N}$. NHC $\mathrm{N}_{6} \mathrm{H}_{5}+\mathrm{H}_{2} \mathrm{O}$.

Dieses Verhalten entspricht vollständig der früberen Beobachtung ${ }^{2}$ ) über die analoge Umwandlung der Methanaldisulfousäure in Methanoltrisulfonsäure.

Phenylhydrazonmethandisulfonsaures Kalium, $\mathrm{C}_{6} \mathrm{H}_{5} \mathrm{NH} \cdot \mathrm{N}: \mathrm{C}\left(\mathrm{SO}_{3} \mathrm{~K}\right)_{2}$.

Man rersetzt das Sulfitadditionsproduct des diazomethandisulfonsauren Kaliums in der 8 fachen Menge Wasser mit überschüssigem (1 $1 / 2$ Mol.), möglichst concentrirtem Diazobenzolacetat und lässt über Nacht stehen. Nach Trennung von den ausgeschiedenen anorganischen Salzen wird die entstandene Schwefelsäure mit Baryumacetat genau ausgefällt und bis zur beginnenden Krystallisation eingeengt. Nach dem Erkalten wird filtrirt und das Hydrazon aus dem Filtrat durch

1) Diese Berichte 28, 1694.

$\Rightarrow$ Diese Berichte 28, 2380 . 
Alkobol gefällt. Es wird aus heissem Wasser, eventuell unter Zusatz ron Alkohol umkrystallisirt.

Analyse: Ber. für $\mathrm{C}_{7} \mathrm{H}_{6} \mathrm{~N}_{2} \mathrm{~S}_{2} \mathrm{O}_{6} \mathrm{~K}_{2}$.

$$
\begin{aligned}
& \text { Procente: C 23.6, Н } 1.7, \quad \mathrm{~N} 7.9, \quad \mathrm{~K} 21.9 \text {. }
\end{aligned}
$$

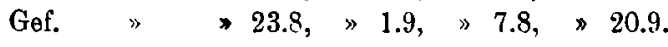

Das Salz, welches übrigens zweckmässiger aus der unten beschriebenen Diazoamidoverbindung durch Spaltung mit verdünnter Salzsăure dargestellt wird, krystallisirt aus heissem Wasser oder verdünntem Alkohol in glänzenden flachen Nadeln. Die Lösung in concentrirter Schwefelsäure wird durcb Ferrichlorid oder Kaliumchromat violet. Beim Kochen mit Säuren tritt Spaltung ein, es wurden Phenylhydrazin und schweflige Säure nachgewiesen. Salpetrige Säure liefert ein mit Wasserdämpfen füchtiges, aus Wasser in feinen Nadeln krystallisirendes, scbwefelfreies Product rom Schmp. $88-90^{\circ}$. Diazobenzol kuppelt in alkalischer Lösung zu der folgenden Verbindung.

Diazobenzolphenylhydrazonmethandisulfonsaures Kalium, $\mathrm{C}_{6} \mathrm{H}_{5} \mathrm{~N}: \mathrm{N} . \mathrm{N}\left(\mathrm{C}_{6} \mathrm{H}_{5}\right) \cdot \mathrm{N}: \mathrm{C}\left(\mathrm{SO}_{3} \mathrm{~K}\right)_{2}$.

Zur Darstellung gebt man besser vom sulfohydrazimetbandisulfonsauren Kalium aus. $42 \mathrm{~g}$ Anilin werden in $200 \mathrm{~g}$ Wasser und $100 \mathrm{~g}$ rauchender Salzsäure diazotirt und bei $-5^{0}$ in eine Auflösung von $53 \mathrm{~g}$ Aetzkali in $250 \mathrm{~g}$ Wasser gegossen. Diese Mischung lässt man in eine Lösung ron $80 \mathrm{~g}$ der Sulfitverbindung in $500 \mathrm{~g}$ Wasser fliessen, worauf unter Aufschäumen die Diazoamidoverbindung als orangegelber Niederschlag ausfällt, der durch Potasche völlig ansgesalzen und alsbald abgesaugt wird. A usbeute $60 \mathrm{~g}$.

Zur Reinigung rübrt man mit der doppelten Menge Wasser an, saugt ab, behandelt das Ungelöste nun mit der 12 fachen Menge Wasser und fält die vereinigten Filtrate mit Alkobol.

Analyse der vacuumtrocknen Substanz: Ber. für $\mathrm{C}_{13} \mathrm{H}_{10} \mathrm{~N}_{4} \mathrm{~S}_{2} \mathrm{O}_{6} \mathrm{~K}_{2}$.

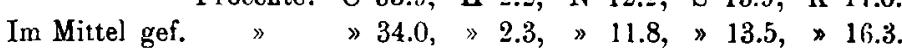

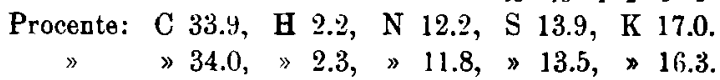

Orangegelbe feine Nädelchen, die sich am Licht dunkler färben. Mit gelber Farbe in reinem Wasser leicht löslich, schwerer in alkaljhaltigem. Verpuffen im Schmelzröhrchen bei $180^{\circ}$. Concentrirte Scbwefelsäure löst mit violetter Farbe. Quecksilbernitrat verursacht einen purpurnen Niederschlag. Alkalische Reductionsmittel färben vorübergebend intensiv roth.

Durch kalte verdünnte Säuren wird die Lösung der Diazoamidoverbindung entfärbt und entbält dann Hydrazon und Diazolenzol. Versetzt man nun mit Kali, so entsteht wieder ein Niederschlag des Diazoamidosalzes, Alkobol fällt dagegen Hydrazon aưs ( 2 wecknässige Darstellung desselben). Alkalisches $p$-Naphtol liefert 
die theoretische Menge Azofarbstoff. Beim Kochen mit Säuren entweicht Diazostickstoff.

$$
\begin{gathered}
\text { Analyse: Ber. Procente: N } 6.1 . \\
\text { Gef. }
\end{gathered}
$$

Bemerkenswertb ist das Verhalten der wässrigen neutralen oder alkalisch gemachten Lösung. Schon bei gewöbnlicher Temperatur, schneller in der Wärme, fallen schwefelfreie Nädelchen vom Schmp. 1870, die noch nicht näher untersucht wurden, aus, während in der Lösung zwei Verbindungen enthalten sind, nämlich das vorstehend beschriebene Hydrazon und benzoldiazosulfonsaures Kalium. Zur Trennung dampft man bis zur Krytallisation ein, worauf beim Erkalten die Diazoverbindung auskrystallisirt; aus dem Filtrat fällt Alkohol das Hydrazon.

Das phenylhydrazonmethandisulfonsaure Kalinm wurde durch Eigenschaften und Analyse identificirt:

Analyse: Ber. für $\mathrm{C}_{7} \mathrm{H}_{6} \mathrm{~N}_{2} \mathrm{~S}_{2} \mathrm{O}_{6} \mathrm{~K}_{2}$.

Procente: C 23.6, H 1.7, N 7.9, S 18.0, K 21.9.

Gef. $\gg \quad \gg 23.6, \gg 1.7, \gg 8.0, * 18.0, \geqslant 21.8$.

Das benzoldiazosulfonsaure Kalium besass Eigenschaften und Zusammensetzung dieser Verbindung:

Analyse: Ber. für $\mathrm{C}_{6} \mathrm{H}_{5} \mathrm{~N}_{2} \mathrm{SO}_{3} \mathrm{~K}$.

Procente: C 32.1, H 2.0, N 12.5, S 14.2, K 17.4.

Gef. 》 "33.0, "1.9, "12.7, 》14.1, 》17.2.

Seine Entstehung ist erklärlich, weil seine Componenten, Diazobenzol und Kaliumsulfit, unter den Zersetzungsproducten der Diazoverbindungen durch Wasger oder Alkalien auftreten.

Alkoholische Salzsäure lagert um zu

$$
\text { Formazylsulfosäure, } \mathrm{SO}_{3} \mathrm{H} . \mathrm{C} \begin{aligned}
& \mathrm{N} . \mathrm{NHC}_{6} \mathrm{H}_{5} \\
& \mathrm{~N}: \mathrm{NC}_{6} \mathrm{H}_{5}
\end{aligned} \text {. }
$$

Die Diazoamidoverbindung wird fein zerrieben, in der 10 fachen: Menge Alkohol suspendirt und unter Kühlung mit alkoholischer Salzsäure versetzt, bis ein Brei von violetten stark reflectirenden Schuppen. entstanden ist. Abgesaugt, auf Thon getrocknet und aus viel knchendem Essigätber umkrystallisirt.

Analyse: Ber. für $\mathrm{C}_{13} \mathrm{H}_{12} \mathrm{~N}_{4} \mathrm{SO}_{3}$.

Procente: C 51.3, H 3.9, N 18.4, S 10.5 .

$$
\text { Gef. 》 \50.9, 》4.3, 》18.3, 》 } 10.7 \text {. }
$$

Violette, blau reflectirende Blättchen, schwer löslich in den meisten Lösungsmitteln. Sintern im Schmelaröhrchen bei 1920. Concentrirte Schwefelsäure löst mit blauer Farbe, welche durch etwas Salpetergäure in Violet umschlägt. Beim Kochen mit verdünnten Säuren wird der Körper zerstört, wobei schweflige Säure und Phenylhydrazin nachgewiesen wurden. Reductionsmittel entfärben fast augenblicklich; da- 
bei entstehen Anilin, Pbenylhydrazin (nicht immer nachweisbar) und merkw ürdigerweise pbenylbydrazonmethandisulfonsaures Kalium, dessen Bildung schon oben erklärt wurde. Zur Identificirung wurde es analysirt:

Analyse: Ber. für $\mathrm{C}_{7} \mathrm{H}_{6} \mathrm{~N}_{3} \mathrm{~S}_{2} \mathrm{O}_{6} \mathrm{~K}_{2}$.

Procente: N 7.9, K 21.9.

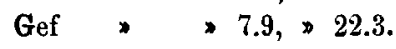

Formazylsulfonsaures Kalium krystallisirt aus der Lösung der Säure in Kaliumacetat in rothen, cantharidenglänzenden Nadeln, die aus heissem Wasser oder sehr viel kochendem Alkohol umkrystallisirt werden können.

$p$ - Bromdiazobenzol phenylbydrazon methandisulfonsaures $\mathrm{Kalium}, \mathrm{C}_{6} \mathrm{H}_{4} \mathrm{BrN}: \mathrm{N} . \mathrm{N}\left(\mathrm{C}_{6} \mathrm{H}_{5}\right) \mathrm{N}: \mathrm{C}\left(\mathrm{SO}_{3} \mathrm{~K}\right)_{2}$.

Aus dem Hydrazon und $p$-Bromdiazobenzol in Potasche-alkalischer Lösung. Orangegelbe, relativ schwer lösliche Nädelchen; zeigen das Verhalten des bromfreien Salzes.

Analyse: Ber. für $\mathrm{C}_{13} \mathrm{H}_{9} \mathrm{BrN}_{4} \mathrm{~S}_{2} \mathrm{O}_{6} \mathrm{~K}_{2}$.

Procente: $\mathrm{Br} 14 . \mathrm{S}$.

Gef. " " 15.0 .

$p$-Bromformazylsulfonsäure, $\mathrm{SO}_{3} \mathrm{H} . \mathrm{C}$ N $: \mathrm{NHC}_{6} \mathrm{H}_{3}$

Aus vorstehender Verbindung und alkoholischer Salzsäure. Violette Schuppen aus Essigäther. Sintern bei $196^{\circ}$.

Analyse: Ber. für $\mathrm{C}_{13} \mathrm{H}_{11} \mathrm{BrN}_{4} \mathrm{SO}_{3}$.

Procente: $\mathrm{Br} 20.9$.

Gef. $\quad$ » 20.9 .

Benzoylhydrazone und Diazobenzol.

Obwobl das verschiedene Verbalten des Diazobenzols einerseits gegen Phenylhydrazone (Bildung von Formazylverbindungen), andrerseits gegen die Sulfitadditionsproducte aliphatischer Diazokörper (wobei zunächst Hydrazone entstehen), darauf hinweist, dass letztere nicht die Constitution von Hydrazonen besitzen können, so war es doch wünschenswerth, auch das Verhalten eines Hydrazons kennen zu lernen, welches nicht vom Phenylhydrazin, sondern einem Hydrazin abstammt, welches durch aliphatischen Kohlenstoff substituirt ist. Zu diesem Zwecke wurden das Benzoylhydrazon des Benzaldebydes $\left.{ }^{1}\right), \mathrm{C}_{6} \mathrm{H}_{5} \mathrm{CONH} . \mathrm{N}: \mathrm{CHC}_{6} \mathrm{H}_{6}$, und der Brenztraubensäure, $\mathrm{C}_{6} \mathrm{H}_{5} \mathrm{CONH}$. N : $\mathrm{C}\left(\mathrm{CH}_{3}\right) \mathrm{COOH}$, gewählt.

Ersteres wurde unter den Bedingungen eines zweimaligen Versuches von Diazobenzol fast gar nicht angegriffen; letzteres lieferte

1) Curtius, Journ. für prakt. Chem. 50, 301. 
- es kam dabei $p$-Nitrodiazobenzol zur Anwendung - unter Abspaltung von Brenztraubensäure eine Verbindung von der muthmaasslichen Formel:

$$
\mathrm{C}_{6} \mathrm{H}_{5} \mathrm{CONH} . \mathrm{NH} . \mathrm{N}: \mathrm{N} \mathrm{C}_{6} \mathrm{H}_{4} \mathrm{~N} \mathrm{O}_{2},
$$

welche auch aus Benzoylhydrazin entsteht und demnach ein Analogon des ron Curtius ${ }^{1}$ ) entdeckten , Hippurylphenylbuzylens ist. Da der Körper nicht umkrystallisirbar ist, können $\mathbf{z}$ war keine analytischen Belege dafür erbracht werden, an jener Formel ist aber kaum zu zweifeln, besonders weil beim Erwärmen mit irgend einem Lösungsmittel der Hauptsache nach Spaltung in Benzamid und $p$-Nitrodiazobenzolimid stattfindet:

$$
\mathrm{C}_{6} \mathrm{H}_{5} \mathrm{CONH} . \mathrm{NH} . \mathrm{N}: \mathrm{NC}_{6} \mathrm{~B}_{4} \mathrm{NO}_{2}=\mathrm{C}_{6} \mathrm{H}_{5} \mathrm{CONH}_{2}+\stackrel{\mathrm{N}}{\ddot{\mathrm{N}}}>\mathrm{NC}_{6} \mathrm{H}_{4} \mathrm{NO}_{2} \text {. }
$$

Das Benzoylhydrazon der Brenztraubensäure wird demnach zwar durch Diazobenzol wie die Sulfitderivate der aliphatischen Diazoverbindungen gespalten; eine Anologie in der Constitution jener Körper kann aber daraus nicht gefolgert werdeu, weil der weitere Verlauf der Reaction sich verschieden gestaltet, indem das Diazobenzol im ersteren Falle an das Hydrazin, in dem letzteren dagegen an das Keton tritt.

$$
\begin{gathered}
\text { Benzoyl hydrazonbrenzt raubensäure, } \\
\mathrm{C}_{6} \mathrm{H}_{5} \mathrm{CONH} . \mathrm{N}: \mathrm{C}\left(\mathrm{CH}_{3}\right) \mathrm{COOH} \text {. }
\end{gathered}
$$

Verdüante reine Brenztraubensäure wird mit der beissen wässrigen Lösung des äquivalenten Benzoylhydrazins vermischt und das nach dem Erkalten auskrystallisirte Hydrazon aus kochendem Wasser oder verdünntem Alkohol umkryatallisirt.

Farblose Nadeln, die $1 \mathrm{Mol}$. Wasser enthalten und bei $112^{\circ}$ schmelzen; bei $110-115^{\circ}$ oder bei längerem Liegen im Vacuum über Schwefelsäure geben sie Wasser ab, worauf der Schmelzpunkt auf $155^{\circ}$ steigt.

Analyse: Ber. für $\mathrm{C}_{10} \mathrm{H}_{10} \mathrm{~N}_{2} \mathrm{O}_{3}, \mathrm{H}_{2} \mathrm{O}$.

$$
\begin{array}{cccc}
\text { Procente: } & \mathrm{N} 12.5, & 1 \mathrm{H}_{2} \mathrm{O} & 8.0 . \\
\text { Gef. } &
\end{array}
$$

In Alkalien und Alkalicarbonaten ohne Zersetzung löslicb. Bemerkenswerth ist die Leichtigkeit, womit die Brenztraubensäure nicht nur durch Diazobenzol, sondern auch durch Benzaldebyd schon in der Kälte verdrängt wird.

$$
p \text {-Nitrodiazobenzolbenzoylbydrazin }
$$

entsteht sowohl aus dem oben beschriebenen Hydrazon als aus Benzoylbydrazin und Nitrodiazobenzol in essigsaurer Lösung. Citronengelber

1) Diese Berichte 26, 1268 . 
Niederschlag, der beim Erhitzen mit Wasser oder Alkobol in Benzamid und das Griess'sche

$$
p \text {-Nitrodiazobenzolimid }
$$

zerfällt, welch letzteres analysirt wurde.

Analyse: Ber. für $\mathrm{C}_{6} \mathrm{H}_{4} \mathrm{~N}_{4} \mathrm{O}_{2}$.

$$
\begin{aligned}
& \text { Procente: C 43.9, H 2.4, N 34.1. }
\end{aligned}
$$

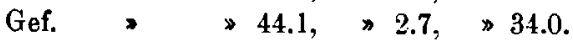

Gelbliche Blättchen aus Ligroïn, Schmp. $71-72^{\circ}$, mit Wasserdämpfen flüchtig. Entstebt glatt auch aus Hydrazin and Nitrodiazobenzol in essigsaurer Lösung neben Ammoniak.

Vorstehende Untersuchung, welche durch die Schwierigkeiten, denen wir anfänglich bei der Reindarstellung der beschriebenen schwefelbaltigen Verbindungen begegneten, nicht mübelos war, wurde von Hrn. Dr. Oscar Unger mit unermüdlicher Geduld ausgefübrt, wofür ich ihm auch an dieser Stelle meinen besten Dank ausspreche.

405. W. Autenrieth: Berichtigung.

(Eingegangen am 15. August.)

In No. 11 dieser Bericbte habe ioh mitgetheilt, dass ans den beiden isomeren $\beta$-Chlorcrotonsäureätbylestern mit Phenylhydrazin unter den gleichen Bedingungen auch die gleichen Reactionsproducte entsteben, nämlich Phenylmethylpyrazolon (Schmp. 127 U), Bisphenylmethylpyrazolon undeinein rotben $\mathrm{Nadelnkrystallisirende}$ Substanz vom Schmp. $155^{\circ}$. Diese letztere Substanz bat sich als identisch erwiesen sowobl mit dem von $\mathrm{Knorr}^{1}$ ) aus Diazobenzolchlorid und Phenylmethylpyrazolon erbaltenen 1-Phenyl-3-methylpyrazolon-4-azobenzol, als auch mit dem von demselben Forscher aus Rubazonsäure und Phenylhydrazin gewonnenen Phenylhydrazin-1-phenyl-3-methyl-4-keto-5-pyrazolon; hierdurch war aber natürlich auch der Nachweis der Identität der beiden letzteren Substanzen erbracht. Hr. Prof. Knorr hat die Freundlichkeit gehabt, mir mitzutheilen, dass ich eine hierauf bezügliche Abhandlung von ihm ${ }^{2}$ ) überseben babe, in welcber er diesen Nachweis schon gebracht und erwähnt hat, dass die fragliche Substanz vom Schmp. $155^{\circ}$ als das Phenylhydrazon des Phenylmethyl-4-ketopyrazolons anzuseben sei.

In der erwähnten Abhandlung habe ich angeführt, dass das Bis. phenglmethylpyrazolon auf Grund von mir dargestellter Acidyl-

1) L. Knorr, Ano. d. Chem. 238, 137.

9) L. Knorr, diese Berichte 21, 1201. 